\title{
Dynamic Contact Network Simulation Model Based on Multi-Agent Systems
}

\author{
Fatima-Zohra Younsi, University of Oran 1, Ahmed BenBella, Algeria \\ Djamila Hamdadou, University of Oran 1, Ahmed BenBella, Algeria
}

\begin{abstract}
Epidemic spread poses a new challenge to the public health community. Given its very rapid spread, public health decision makers are mobilized to fight and stop it by setting disposal several tools. This ongoing research aims to design and develop a new system based on multi-agent system, suscpetibleinfected-removed (SIR) model, and geographic information system (GIS) for public health officials. The proposed system aimed to find the real and responsible factors for the epidemic spread and explaining its emergence in human population. Moreover, it allows to monitor the disease spread in space and time and provides rapid early warning alert of disease outbreaks. In this paper, a multiagent epidemic spread simulation system is proposed, discussed, and implemented. Simulation result shows that the proposed multi-agent disease spread system performs well in reflecting the evolution of the dynamic disease spread system's behavior.
\end{abstract}

\section{KEYWORDS}

Epidemic Dynamics, Geographic Information System, Multi-Agents System, Risk Graph, SIMAS System, Simulation, Susceptible-Infected-Removed Model

\section{INTRODUCTION}

Infectious diseases are worrying, increasingly, authorities and public health officials. Despite continuous and effective monitoring of infectious diseases, it should be noted that their etiology remains unrecognized amplification.

To date, few information systems have been developed to help public health officials in the analysis of multidisciplinary data (Younsi et al., 2017). The epidemiological surveillance systems developed aim various objectives, such as: monitoring, surveillance, prevention, alert, etc. Indeed, some researchers consider these systems as decision support tools in the field of epidemiological disease prevention and control (Toma et al., 1991). However, in our opinion, these systems suffer from deficiencies related to the analytical aspects as well as their limits to deal with several actions at once, such as: monitoring, prevention and alert. In this context, some problems related to the spread of infectious diseases are complex in nature and involve many factors obtained from several fields, qualitative and/or quantitative in nature. These factors are considered as the main causes of the spread of many diseases among the human population. Indeed, the problems related to the spread of infectious diseases are multiple and dependent on many factors that need to be analyzed in a global dimension. Among these factors characteristics and dynamics of the disease (mode of transmission, duration of infections, etc.), the social structure of individuals such as the concentration of the population in urban areas and individual exchanges and encounters, socio-economic factors, etc. 
Tracking dynamics of infectious diseases and detecting changes in a disease process are impossible without development and implementation of mathematical and social methodology dedicated to spatiotemporal disease surveillance. Mathematical and simulation models are, increasingly, used to study the transmission of infectious diseases in order to predict how they might spread in a human population during pandemic event, to evaluate different intervention strategies and to help public health decision makers with disease outbreak control and management (Younsi et al., 2019a). Various mathematical models existing in literature can be used to represent the different state of patients (compartment) in the course of the illness (S: susceptible, I: infective, R: removed, etc.). These deterministic models assume that populations are completely mixed and ignore spatial effects of spread epidemics, also interaction between individuals is neglected since they model populations as continuous entities (Perez and Dragicevic, 2009). In this study, we used the Susceptible, Infected, Removed (SIR) model within Small World network (SW) to explore and understand the dynamics of flu epidemic.

Epidemic transmission occurs through person-to-person contact, and contact between susceptible and/or infected individuals takes the form of a network. Moreover, the complex nature of human life (its relations contacts forming a complex human network, its commuting, its socio-economic life, etc.) makes it difficult to understand how changes will affect the incidence. Unlike to compartment models, the agent based model is particularly appropriate in our study. Processes are defined at the individual level, allowing a definition of the patient's history and the complexity of within-host phenomena. In addition, we discuss the effect of the vaccination to show the effectiveness of vaccine at preventing disease and infection.

Geographic Information System (GIS) and Database Management System (DBMS) are, widely, used in the scientific world and are recognized as tools for decision support exploited in various fields who are interested in the study of the territory and its people (Mesgari and Masoomi, 2008). Indeed, the health officials are in the need for a geo-decision-making tool for monitoring and surveillance of epidemic in this network. Herein, we highlight the role of GIS in monitoring epidemic spread by visualizing risk seasonal influenza map.

\subsection{Our Contribution}

In this study, we are interested in the modeling and implementation of a spatiotemporal decision support system based on SIR model using multi-agent model. The main goal of Simulation model for Influenza epidemic based on Multi-Agent System (SIMAS) is to ensure a well-considered management of the epidemic spread and to be able to evaluate prevention strategies and identify the leading risk factors that put persons at increased risk. Moreover, it is important to design and set up a quality and efficiency monitoring system for outbreaks spread. The proposed system allows the simulation of the phenomenon of the epidemic spread in a human population in order to provide public health decision-makers an epidemiological monitoring and prevention tool.

Indeed, in our research, we design and develop a new model based on compartment epidemic model and social network model called Susceptible-Infected-Removed within Small World network (SIR-SW) model. The SW model adapts and extends the models for networks of social contacts proposed by (Watts and Strogatz, 1998). The simulation system generates a number of susceptible, infected and removed individuals in time and space Indeed, we are not only interested by modeling driven approach that involves coupling models from different scientific fields (SIR model and SW model), but also with multi agent-driven approach that is compatible with compartmental epidemic models where the human population, modelled as agents, behaves and interacts in a small world network and the gathering place (work, school, household, etc.) are considered.

In other words, our purpose is to design, model and develop a spatiotemporal decision support system SIMAS for seasonal influenza data collected by the Public Health Department of Oran $\left(\mathrm{PHD}^{1}\right)$ for 26 locations in Oran city (Algeria). Over this period, seasonal influenza was the most 
frequently strain, the collected data concern it. The proposed model represents some hypothesizes about transmission dynamics.

Other specific objectives of the current study are:

- Model and simulate the phenomenon of the influenza epidemic spread in a human population by using a multi-agent approach;

- Identify the main factors promoting the influenza transmission;

- Study in time and space the global and local behavior of individuals;

- Visualize on a geographic map the different risk areas of flu epidemic.

The proposed system SIMAS is mainly dedicated to the surveillance and prevention of the seasonal influenza epidemic. It is designed by gathering three main components:

1. The Multi-Agent System allows to take into account individual behaviors, interactions between individuals and dynamic hypotheses formulated;

2. The epidemic propagation system (simulation system) is based on the Susceptible-InfectedRemoved (SIR) compartment model. It enables to explain and control the evolution of seasonal influenza in a human population through simulations;

3. The visualization system enables to present the risk areas of flu epidemic on the map of Oran city (Algeria). Moreover, the SIMAS system allows, also, to visualize the social network of individuals during the infection by using the Watts and Strogatz approach (Watts and Strogatz, 1998).

The remainder of this paper is organized as follows: Section 2, briefly overviews related works. Section 3 describes the proposed system. In section 4, we outline the UML Use Case Diagram modeling SIMAS and the adopted decision making process. Section 5 presents the obtained results. Finally, Section 6 concludes the paper.

\section{RELATED WORKS}

Epidemiological prevention is considered necessary for the protection of human health. Evaluation of control activities, early detection of emergent phenomena and decision on control methods to be used should be based on reliable monitoring data and recognized. In this paper, we focus on two main types of approaches discussed in the following.

\subsection{Multi-Agent Simulation System Using Complex Networks}

In the context of epidemiology, multi-agent simulation systems are frequently used. In what follows, we discuss the most relevant to our research.

In Cao et al. (2008), the authors propose a mobile multi-agent system based on Vicsek model for epidemic dynamics. In their model, mobile agent contacts with his spatial neighbors with the Susceptible-Infected-Susceptible (SIS) epidemic mechanism. The numerical simulation shows that the strategy can improve the epidemic threshold, which is therefore valid for epidemic control. Using the Multi-Agent System (MAS) within geo-spatial environment to build the simulation model, SIQR epidemic model is introduced to simulate the process of the spread of influenza A (H1N1) in Xiao et al.(2010). The testing results indicate that: the control of short-term epidemic spread requires administrators to implement powerful effective measures in public places to isolate patients and the number of the immune and the susceptible are negatively correlated. The authors in Dong et al.(2012) studied the spread of flu in small population by collecting data about the individuals' daily symptoms. The authors were able to fit the collected symptom data to SIS like-disease. Such study 
gives insights about the relationship between the proximity of individuals and the spread of flu. An evolutionary game and simulation model based on the interactions between strategies and states was proposed in Yang et al. (2015). The game between the public and government sectors and its impact on epidemic situations was discussed. For the analysis replicator dynamics equations and the multiagent model simulation were used. Zhang et al. (2016) described a way to model relatively complete spatial contacts in the context of a large-scale artificial city, which combines different data sources to construct an agent-based model of the city Beijing. The simulation results shows potential not only for improving comprehension of disease spread dynamics, but also for use in other social systems, such as public transportation systems and city level evacuation planning. A novel Agent-Based Model $(\mathrm{ABM})$ of influenza transmission during interaction with another respiratory pathogen was developed in Arduin et al.(2017). In this work, different mechanistic hypotheses of interaction are simulated and the resulting virus-induced pneumococcal infection (PI) burden was assessed. The aim of Hackl and Dubernet(2019) study is to propose a large-scale agent-based transport simulation (MATSim) which is linked with a generic epidemic spread model to simulate the spread of communicable diseases in an urban environment. The results of disease propagation simulation indicate that the model is successfully able to generate various scenarios of an outbreak in complex and realistic urban settings by incorporating movement in the agent entities. Recently, the authors in Kano et al.(2021) propose an agent-based model of the COVID-19 outbreak that accounts for economic activities. The computational simulation of the model recapitulates the trade-off between the health and economic damage associated with voluntary restraint measures. Their result suggest that in real societies, governments should make efforts to reduce the economic gap; otherwise, voluntary restraint measures cannot continue, which makes it difficult to mitigate an outbreak.

The list above is certainly not exhaustive, but rather provides representative literature related to our research. Moreover, there are many references in the literature allowing the use multi-agent systems in the context of epidemiology.

\subsection{Epidemiological Surveillance Systems}

The evolution of epidemiological surveillance system has affected several levels: administrative (political actors, directors, etc.), medical (doctors, epidemiologists, etc.), etc.

In the literature of epidemiological surveillance, we find several systems either on a national level like sentinel networks (France, Belgium, United States, United Kingdom, Sweden, etc.), the National Institute for Public Health Surveillance (INVS), etc. or, on a global level like the American system LEADERS (Lightweight Epidemiological Advanced Detection and Emergency Response System) (Ritter, 2002), it is a national system which assists in tracking outbreaks, and helps coordinate large scale real time crisis management. The RODS system (Real-time Outbreak and Disease Surveillance) is developed to collect and analyze disease surveillance data in real time (Moore et al., 2008), $\mathrm{WHO}^{2}$ (World Health Organization), etc. Each system, cited above, is practically different. Thus, each one has its own: goals, working methods, and development tools. Usually, the main purpose of these systems are the calculation of medical statistics, monitoring disease evolution over time and sometimes over space, a periodic publication of statistics, etc. However, these systems are neither based on agent based model to understand the local and global behavior of individual, nor on identification of factors promoting the spread of infectious diseases. Their main aim is monitoring and surveillance, either in time by setting up collection systems to collect data in real-time, or by visualization of risk areas using GIS. Indeed, GIS is used for geospatial data management and analysis. SusceptibleExposed- Infected- and Removed within Small World Network using Geographic Information System (SEIRSW-GIS) system is proposed in Younsi et al.(2015). The proposed system was applied for monitoring epidemic spread. It does not consider agent based model solution for prevention a likely future course of the epidemic. The obtained results of SEIRSW-GIS show its good performances. It becomes essential for any study of epidemiological like alert, monitoring, and surveillance. Other systems based on multi-agent simulation model were developed. 
In Eubank (2005), the author describes an epidemiologic model based on a social network and the Epidemiological Simulation System (EpiSims). Il offers general speculation on analyzing disease dynamics on networks. This system is based on the following principles: the taking into account of individual behaviors, the interactions between individuals, the existence of relational networks and the effects of the spatial structure in the propagation of the epidemic. In Langlois et al. (2015), the authors have developed the MAGéo platform (Modeling Agent in Geography), a user-friendly agentbased simulation tool dedicated to spatialized and multilevel researches. The modeling in MAGéo is, therefore, essentially to build a conceptual diagram consisting of boxes and relationships to organize and then fill in the parameters of the various elements.

Recently, the authors in Godjedo et al. (2020) conducted a retrospective and evaluative study with a mixed approach (qualitative and quantitative) on the three sites identified for sentinel surveillance of meningitis in Benign. All criteria except sensitivity, specificity and positive predictive value were measured. The system performance was recognized with simplicity at $80.8 \%$, good acceptability (completeness: 98.5\%; Promptitude: $88.9 \%$ ) and responsiveness of $85 \%$.

\subsection{Discussion}

The previous researches have led to the development of different models and systems in order to control and prevent epidemic spread in human population. Some researchers used the differential equations to simulate and to control the evolution of epidemic. Nonetheless, such systems fail to understand and analyze the complexity of human behavior and interactions that explain the mechanism of disease spread.

Moreover, the disease transmission in human population is strongly relies on the structure of the underlying social network. Many researches are developed to depict the spatial behavior of diverse infectious diseases through structured and realistic contact networks. Some of the models mentioned represent the spatial distribution and mobility of individuals in order to model the spatial in the disease spread. However, one of their drawbacks is the lack of use of network structures and integration with geographic information systems (GIS) to represent the risk epidemic area.

To our knowledge, no previous studies have treated the spread of epidemic monitoring and prevention context using multi-agent model to understand the local and global behavior of individual, propose a new approach to simulate epidemic spread based on social contact network and SIR epidemic model and visualize the risk area on geographic map and the risk graph to facilitate the decision-making process. Moreover, we highlight the use of the social network analysis to identify the others factors promoting the spread of epidemic.

\section{THE PROPOSED SYSTEM}

In this section, we propose a spatiotemporal decision support system for monitoring and prevention of influenza epidemic (SIMAS). The proposed system responds to public health officials' needs. Indeed, SIMAS is based on three main sub-systems: Multi-agent system, Simulation system based on SIR model and visualization system. An overview of our system is illustrated in Figure 1.

\subsection{Multi-Agent System}

Agent-based models allow interaction among individuals and are capable to overcome the limitations of different approaches such as cellular automata and classical epidemic models, permitting to study specific spatial aspects of the spread of epidemics and addressing naturally stochastic nature of the epidemic process (Perez and Dragicevic, 2009). Modeling in epidemiology using an agent-based approach pursues the progression of a disease through each individual (thus populations become highly heterogeneous by health status during simulations), and tracks the contacts of each individual with others in the relevant social networks and geographical areas. All the rules for individual agent movement (e.g., to and from workplace and/or school) and for contacts with and transmissions to 


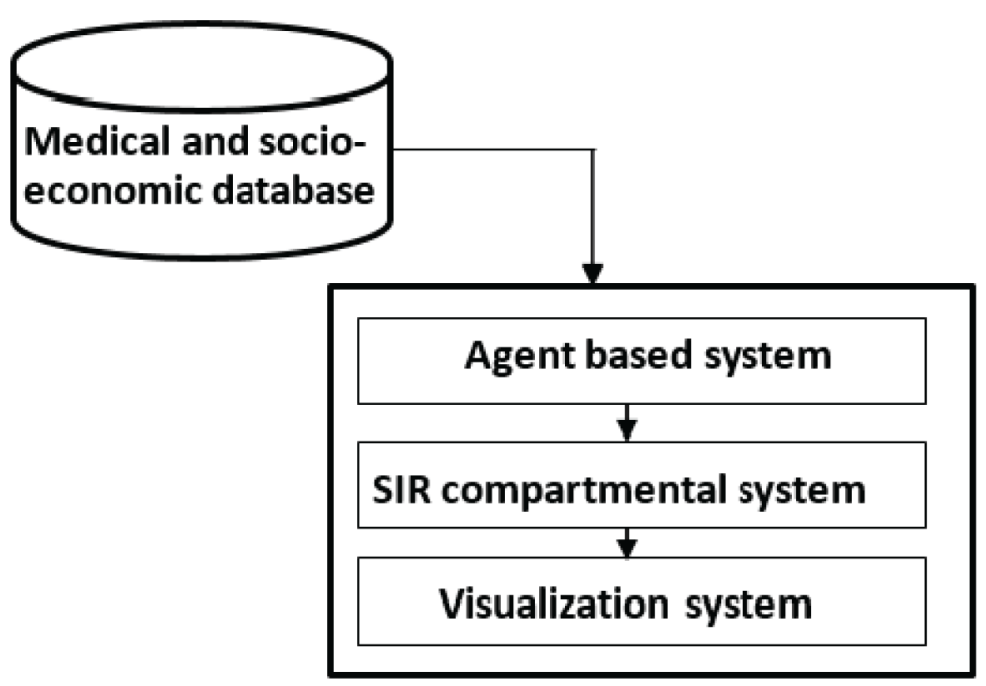

other people are explicit (Epstein et al., 2004). Moreover, agent-based methods simulate individuals and their behavior.

\subsubsection{Description of MAS}

In our multi-agent system, we first create a family network with $\mathrm{N}$ agents ( $\mathrm{N}$ represents the total number of individuals in the host population). $\mathrm{N}$ is divided into 26 communes of Oran:

- An agent: Is defined as an autonomous and communicating entity playing roles within different groups.

- A group: Is composed of a set of agents.

- A role: Represents a function, service or identification of an agent belonging to a particular group. The roles of agents in our work are of two types: initiator (coordinator) and participant: - Initiator: Is the agent responsible for the initiation of the simulation.

- Participant: Is each individual involved in the decision.

We assume that:

1. An agent (individual) will be required to meet at any time a number of other agents.

2. We use cognitive agents because it enables a very good representation of agents' behavior, have a memory (history) and have a goal to achieve collectively.

3. Each agent has an adjacency list (the list of its neighbors). More specifically, it is a list of agents with which he is in contact (interaction). It is important to take into consideration various types of relationships between individuals, which highlights the insufficiency of simple graph modeling.

\subsubsection{Specification of the Agent Behavior}

The social environment is represented by the following structures: Teaching places, Nurseries and Work places.

Each of these structures, considered as a MAS, are located in a geographical area that are composed of a population which represent a set of agents as illustrated by the Figure 2 . 


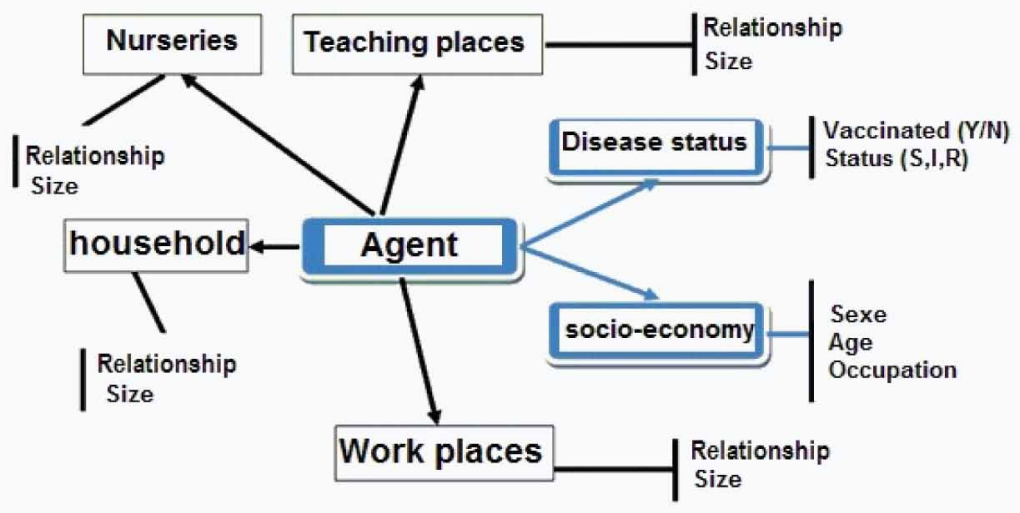

In our world, we have a number of institutions, namely: places of teaching, workplaces or nurseries that are located in geographical areas containing a set of interacting agents. The characteristics of the agents are detailed in the agent model described below.

\subsubsection{Agent Model Specification}

The agent model can be illustrated as shown in Figure 3.

Figure 3. Representation of agent characteristics

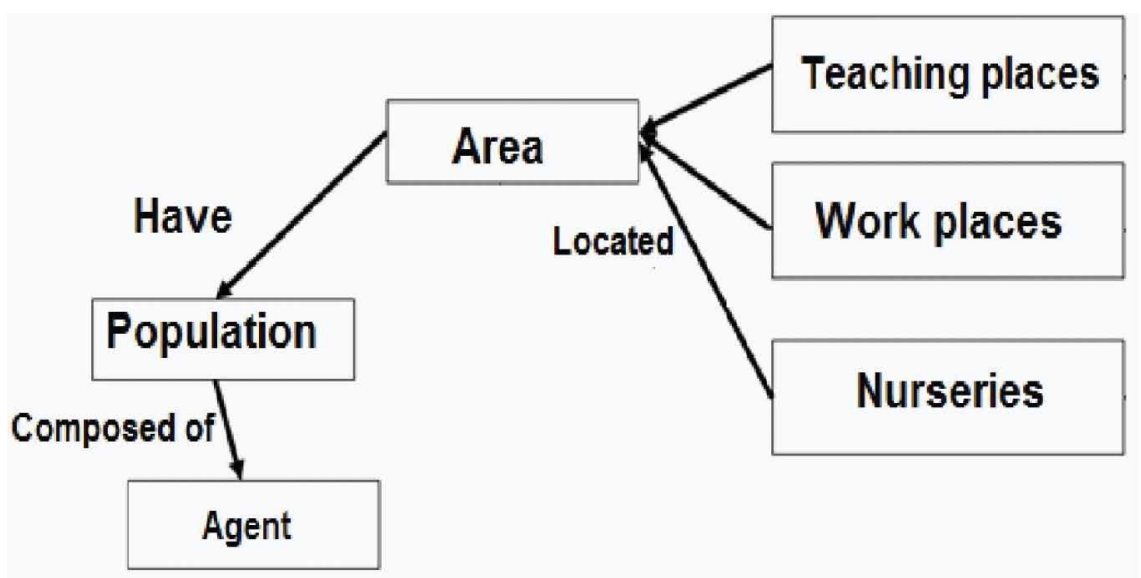

We construct the SW networks using Wattes and Stogatz model which is considered as the typical model of social networks model (Watts and Strogatz, 1998). In the SW network, we generated a set of cognitive agents according to their age and gender. Each agent belongs to a household where the size and the relationship are known.

The agent is characterized by its status during the disease: 
- Vaccinated or not vaccinated: this information is useful because it allows us to judge the immunity of the agent.

- His status of health during the influenza disease: we define the agent status, is the agent in the S (Susceptible), I (Infected) or R (Recovered/retired) status.

We also integrate socio-demographic information about individual. The data was obtained from Public Health Department of Oran (PHD):

- Sex: We have $49 \%$ male agent and $51 \%$ female agent.

- Age: We distinguish four age groups: 0-5, 6-18, 19-59, 60 and over.

- Relationship: The belonging of an agent to a family.

- Type: From the age and sex of each agent, we determine the type of agent: father, mother, child, grandfather, grandmother, etc.

- List of neighborhood: Each agent has a list of agents with whom he is in contact.

- The rate of infection (infection doses): Is a probabilistic value between $[0,1]$ attributed to the agents and allowing the transition of the susceptible state to the infected state.

- The duration of infection: In many studies of epidemiological models, the period of infection is generally assumed to be either an exponential or fixed distribution (Beretta and Kuang, 2001). Here, we consider that it follows an exponential distribution because it is variable from one agent to another.

- Location or residence: Is the district of agent (commune).

It is assumed that the interacting microorganism can persist in the population year round (winter respiratory viruses). As known, single infection with any strain of influenza viruses elicits lifelong antibody-mediated protection against the exact same virus strain (Kilbourne, 1978). According to the PHD of Oran, one strain of flu occurred during the year 2009. This explains the choice of the SIR model. Recovered persons never come back to susceptible class.

\subsubsection{Communication Between Agents (Primitives)}

1. The primitive associated with the Initiator.

Request (): The initiator agent sends this message to the participants to indicate the start of the simulation. Upon receipt of the Request () message by the participants, they must begin the simulation.

2. The primitive associated with the agent.

Inform (): Each infected agent sends this message to the initiator that contains the time of the infection.

\subsection{SIR Compartmental Epidemic Model Within Social Network}

Mathematical epidemic models based on social network are being increasingly used as decision support tools by health officials. In contrast to aggregate differential equation based models, these network models describe diffusion of an infectious disease over a social contact network, which is constructed from individual level demographic and activity data (Xia, 2011). Many researches have shown that almost all realistic networks have small world and/or scale free properties (Kim, 2014). Various networks exist in the literature, in our research, we adopt for SW network introduced by Watts and Strogatz model (Watts and Strogatz, 1998). A SW network is an attempt to represent realistically the network of contacts between individuals, where local links predominate, but social and geographical motilities imply a fraction of random connections through which long-range transmission may occur (Verdasca et al., 2005). 
The SIR model is the basis for all influenza models. It can extend to study more complex situations. In this context, we find the SIR compartmental model incorporating: only treatment and/ or vaccination and treatment and/or quarantine and isolation measures and/or socio-economic and/ or demographic parameters, etc. Therefore, the increase in the performance of the model causes the increase of its complexity (several parameters and state variables).

In SIR compartmental model illustrated in Figure 4, an infectious individual can transmit disease to susceptible individual with probability $\beta$, the susceptible enters the class of infected individuals I and stay infectious for a period of time $\gamma$ ( $\gamma$ is average infectious period). Finally, he is removed and he cannot infect nor be re-infected.

Consider a closed population of individuals (agents), partitioned into three compartments: susceptible (S), infectious (I), and recovered (R). At any time $t=\{0,1, \ldots, T\}$ each agent is a member of exactly one of these three compartments.

Where $\beta>0$ is the disease transmission rate and $\gamma>0$ is the recovery rate.

We denote the proportion of the population in the susceptible, infectious, and recovered compartments by $S(t), I(t)$, and $R(t)$, respectively, such that $S(t)+I(t)+R(t)=N(t)$ where $N$ is the total population.

Figure 4. The standard SIR compartmental model diagram

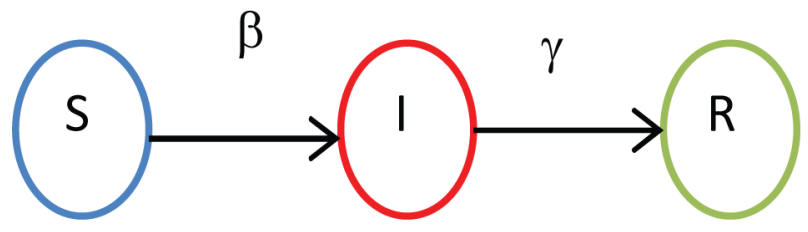

In the SIMAS system, a person interacts with another person when they both visit a same location at the same time (have a direct contact). Indeed, the proposed model concerns only the case where the infection spreads directly between the infected and the susceptible agents (no epidemic vectors). Moreover, the epidemic threshold models indicate that $\mathrm{S}$ agents are not immediately infected when they contact I agents, but after receiving a certain amount of "toxins", namely infection doses, they will be infected (Doddsand Watts, 2004).

All symbols used by the propagation model (simulation system) have their usual meanings as specified in Table 1.

In constructing our model, we consider two kinds of simulation with vaccination and without vaccination. Indeed, we used several key assumptions namely:

- At the beginning, we have a closed and homogeneous agent population partitioned at any time $\mathrm{t}>0$ into three categories: susceptible, infected and recovered. With $\mathrm{N}=\mathrm{S}+\mathrm{I}+\mathrm{R}$ at each unit of time $t$, where $\mathrm{N}$ is the total size of the population and with the initial conditions: $\mathrm{S}(0)=\mathrm{S} 0$, $\mathrm{E}(0)=\mathrm{E} 0, \mathrm{I}(0)=\mathrm{I} 0$ and $\mathrm{R}(0)=\mathrm{R} 0$, respectively.

- During the disease, the agents can have three states: $V=\{$ agent.S, agent.I, agent.R $\}$. Each agent has a list of neighbors who are connected to him.

- $\quad$ Let $I(t)=\{$ agent. $I \in V\}$ is the number of agents infected at this moment.

- Infected agents can transmit the infection to susceptible agents if the cumulative dose which $\mathrm{S}$ agent received exceed a given dose threshold, agent will be infected, otherwise will not be (is defined randomly). 
Table 1. The meaning of all parameters and state variables with units

\begin{tabular}{|l|l|l|}
\hline \multicolumn{1}{|c|}{ List of variables } & \multicolumn{1}{c|}{ Meaning } & \multicolumn{1}{c|}{ Unit } \\
\hline $\mathrm{t}$ & Time & Day \\
\hline $\mathrm{S}(\mathrm{t})$ & Number of susceptible person at $\mathrm{t}$ & Individual (agent) \\
\hline $\mathrm{I}(\mathrm{t})$ & Number of infected person at $\mathrm{t}$ & Individual (agent) \\
\hline $\mathrm{R}(\mathrm{t})$ & Number of recovered person at $\mathrm{t}$ & Individual (agent) \\
\hline $\mathrm{S}_{0}=\mathrm{S}(0)$ & Number of susceptible individual at time $\mathrm{t}=0$ & Individual (agent) \\
\hline $\mathrm{I}_{0}=\mathrm{I}(0)$ & Number of infected individual at time $\mathrm{t}=0$ & Individual (agent) \\
\hline $\mathrm{R}_{0}=\mathrm{R}(0)$ & Number of recovered individual at time $\mathrm{t}=0$ & Individual (agent) \\
\hline $\mathrm{N}$ & Total population & Individual (agent) \\
\hline $\mathrm{k}$ & Average number of neighbors & Individual (agent) \\
\hline$\beta$ & Transmission rate & Rate \\
\hline$\gamma / \gamma^{-1}$ & Recovery rate/ infectious period & Day ${ }^{-1}$ \\
\hline
\end{tabular}

- Each infected agent has a probability of recovery (duration of infection). $\mathrm{I}(\mathrm{t})$ is defined as the list of infected agents and $\mathrm{G}(\mathrm{t})$ as the agent network (or risk graph).

In what follows, we summarize the SIR within SW process without vaccination and with vaccination in a detail pseudo algorithm. The pseudo-algorithms are inspired by the pseudo algorithms used in Younsi et al.(2019b):

\section{BEGIN}

1. Initially, generation of SW network and determination of the list of neighbors of each agent. Then, a number of infected agents are randomly selected. Each simulation starts with only some initially infected agents, all others being susceptible, and it ends when no agent in the infectious state is left;

2. Disease spreads in SW network from infectious agents to susceptible agents if it exists a relationship between them;

3. Each infected agent will be infectious and can transmit infection to other susceptible agents;

4. Each infectious agent will be recover (becomes immune) after an infectious period. After recovery, it cannot transmit the infectious agent again. Its immunity is permanent and it cannot return to the susceptible class;

5. The network $\mathrm{G}(\mathrm{t})$ evolves to $\mathrm{G}(\mathrm{t}+1)$.

The process 2, 3, 4 and 5 are repeated until no infectious individuals exist in the host population. END

The pseudo algorithm of the process with vaccination is described as follows:

\section{BEGIN}

1. Initially, generation of SW network and determination of the list of neighbors of each agent. Then, a number of infected agents are randomly selected. Each simulation starts with only some initially infected agents, all others being susceptible, and it ends when no agent in the infectious state is left;

2. Vaccination of an agent set randomly; 
3. Disease spreads in SW network from infectious agents to susceptible agents who are not vaccinated. If it exists a relationship between them;

4. Each infected agent will be infectious and can transmit infection to other susceptible agents;

5. Each infectious agent will be recover (becomes immune) after an infectious period. After recovery, it cannot transmit the infectious agent again. Its immunity is permanent and it cannot return to the susceptible class;

6. The network $\mathrm{G}(\mathrm{t})$ evolves to $\mathrm{G}(\mathrm{t}+1)$.

The process 3, 4, 5 and 6 are repeated until no infectious individuals exist in the host population. END

\subsection{Visualization System}

During crisis management of public health, decision-makers lack simple and powerful mapping tools to explore, synthesize and analyze spatiotemporal data to identifying areas at risk. Usually, the visualization of outbreaks on geographical maps is managed by a Geographic Information System (GIS).

Herein, SIMAS enables to present the dangerous areas of epidemic on the map of the city of Oran (Algeria). In addition, the system allows to visualize the social network of agents in the form of a circle by referring to the approach of Watts and Strogatz (1998). The SIMAS system, also, enables to calculate and display statistics relating to the evolution of the epidemic in the form of curves or pie charts.

\section{UML AND DECISION MAKING PROCESS}

In what follow, we describe a series of steps that decision makers should consider in the use of SIMAS system. These steps are presented in the form of UML diagram and decision making process.

\subsection{UML Use Case Diagram Modeling SIMAS}

The principal aim of UML Use Case Diagram is to describe behavior of the SIMAS system and a set of actions (use cases) that SIMAS can perform in collaboration with epidemiologist or decision maker and scientist (external users of the system "actors").

The different features provided by our SIMAS system are illustrated by the UML use case diagram presented in Figure 5. Each case-use of the diagram is a system step, there are many usecases and two actors.

\subsection{The Decision-Making Process Adopted by SIMAS}

Decision-making in public health is a complex and dynamic process that is influenced by epidemiology, social, economic and political factors. It provides multiple and iterative analysis, and simulations. In Figure 6, we describe in detail, the decision-making process adopted by SIMAS.

\section{CASE STUDY}

Seasonal influenza causes annual epidemics. It represents a serious public health problem that causes severe disease and death in populations at higher risk. Indeed, the surveillance and monitoring of the influenza epidemic has become a necessity to understand its evolution and occurrence (Younsi et al., 2015).

In this section, we present a case study illustrating the use of SIMAS. First, we describe the influenza disease concerned by this study as well as the study area. Then, we will carry out some numerical simulations. Finally, we discuss visualization model and we display outbreak sources on Oran map. 


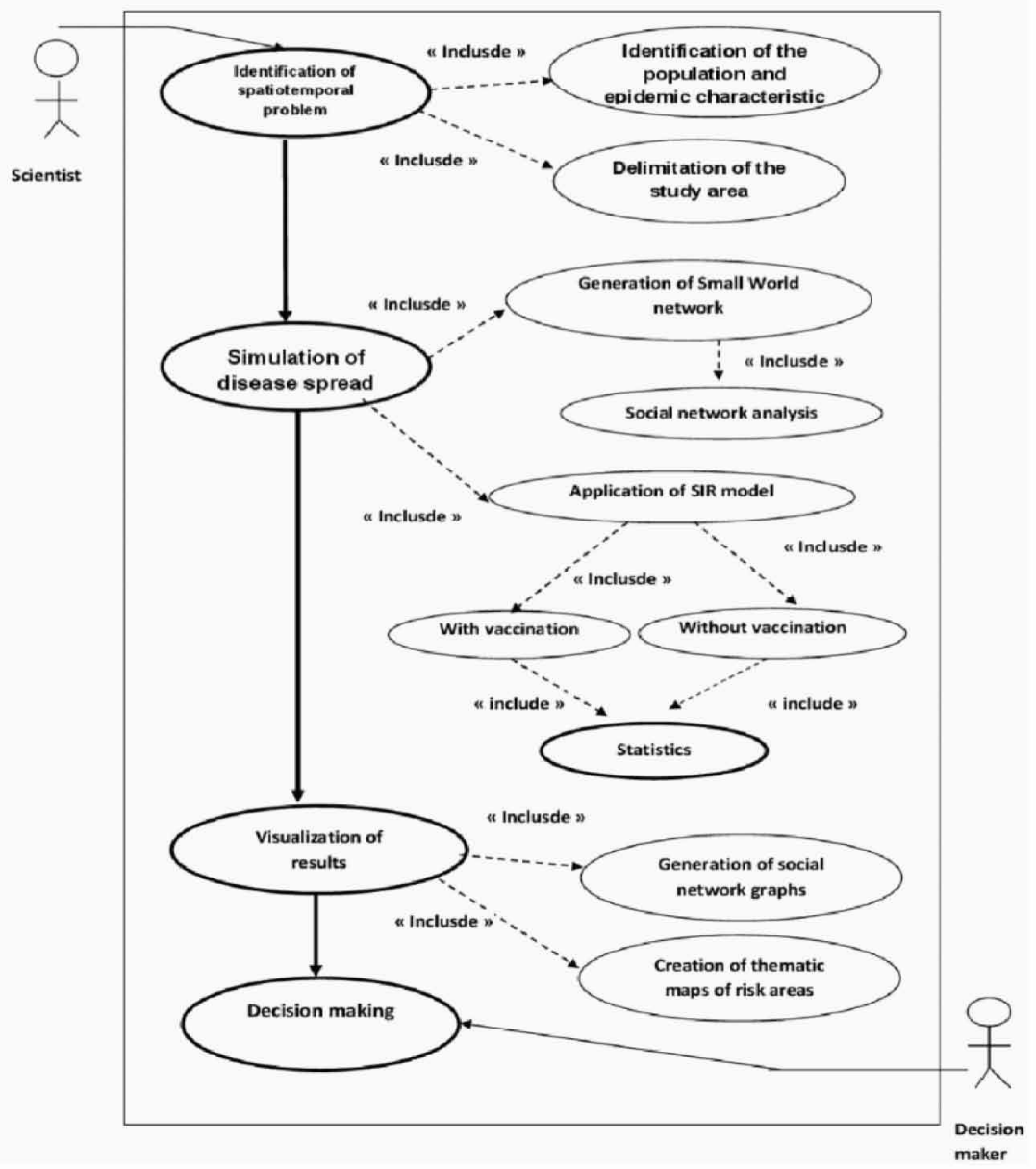

\subsection{Data}

The database used in the current study concerns the seasonal influenza flu (2009) in the region of OranAlgeria. It is a medical and socio-economic database obtained from the Public Health Department (PHD) of Oran. It is composed of 5504 records for the 26 communes (municipalities) of Oran.

The SIMAS model depends on the parameters represented on Table.1. Some parameters are determined from medical and socio-economic data (real data) and others may be obtained from the SIMAS model outcomes fitted to the real data.

\subsection{Description of the Study Area}

The geographical area of interest for this study is the city of Oran- Algeria. This choice is due, mainly, to the importance of this city which is considered the second largest city in Algeria and actually has approximately 1,468,000 inhabitants in an area of $2121 \mathrm{~km}^{2}$. The population of Oran is distributed into 26 communes (municipalities). Figure 7, shows the communes of Oran. 


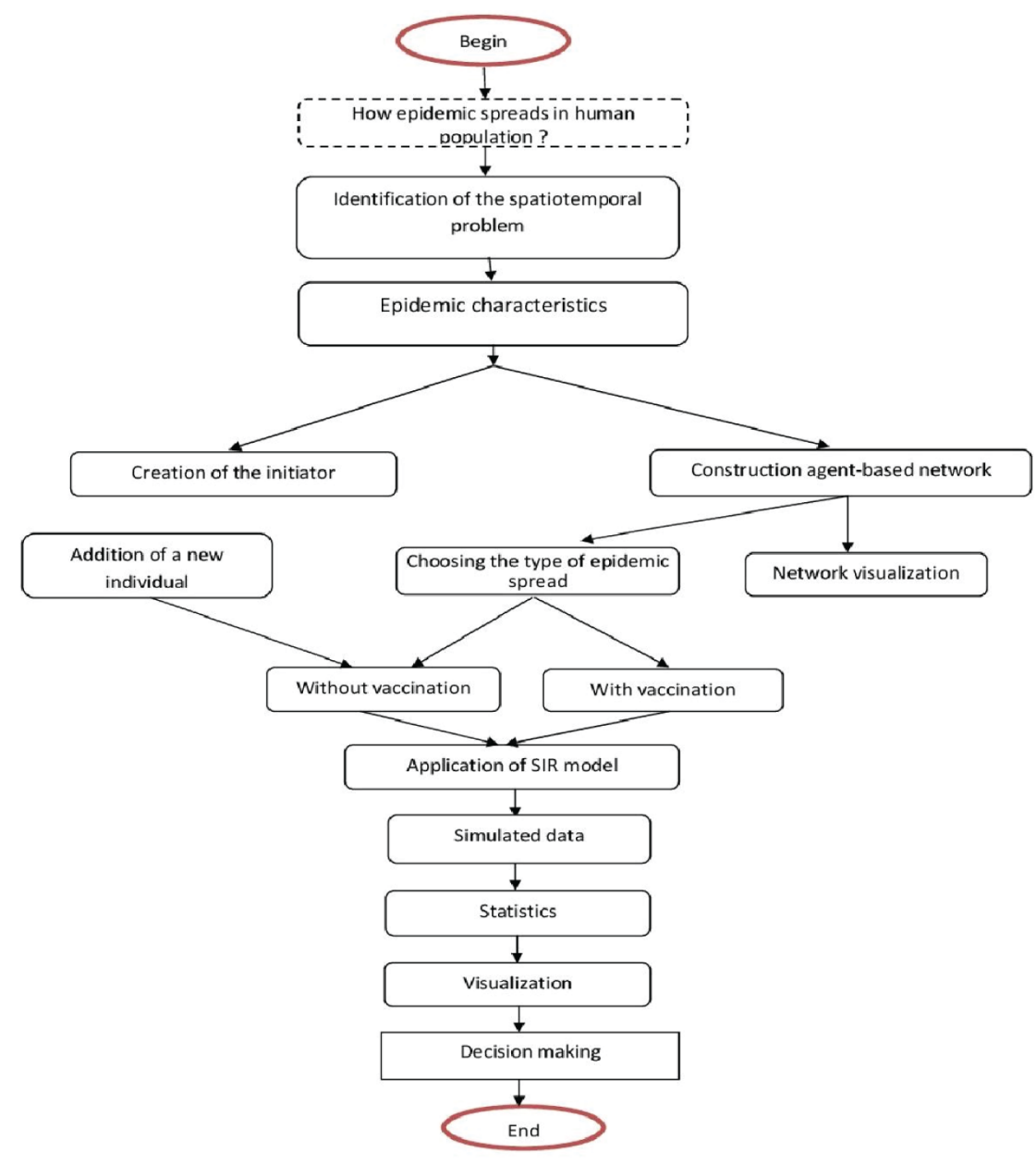

\subsection{Simulation System}

In this section, we outline and discuss the numerical results of simulation influenza epidemic model based on Muti-Agent System and GIS.

We use a compartmental epidemic SIR model to simulate epidemic spread on contact network based on SW model. The latter is composed from agents. The information according to each agent includes name, job, disease status, relationship, residence, etc.

In our model, we will consider influenza flu for short duration relative to human population dynamics, we assume that the population is close i.e. with no birth and no death. Before launching the SIMAS system, the decision maker should set the initial parameters (see Table.2) for simulation and he should, also, define the type of simulation (with or without vaccination). The initial parameters and the selected area of study constitute the inputs of the SIMAS. Decision maker can run the simulation for one commune, some communes or for all communes.

We used the JADE (Java Agent Development Framework) software development framework aimed at developing multi-agent programmable modeling environment for the implementation and 


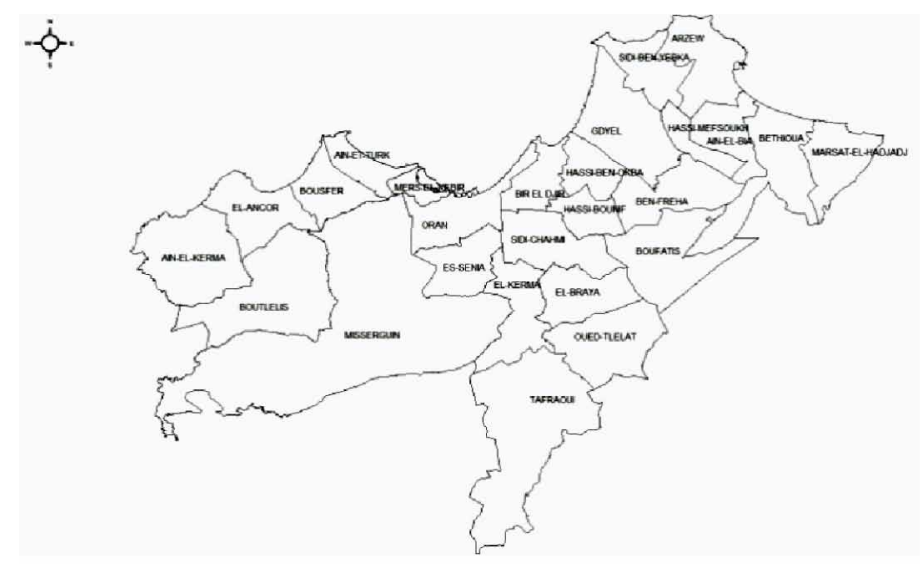

the Sniffer which is completely integrated in JADE environment. The Sniffer is, particularly, useful for tracking messages exchanged in a JADE based environment.

The simulation model will run for each set of parameters and state variables, once the simulation is completed, data outputs are created according to the type of simulation selected. The simulation data are used to generate map outputs for a geo-location of epidemic outbreaks and other simulations statistics.

We have applied simulation model to the 26 communes of Oran with two type of simulation (with and without vaccination) to demonstrate the effectiveness of vaccine, as outcomes of SIMAS system, we have obtained 26 results. Herein, we are interested by three communes that represent a hot area of outbreak flu: commune of Oran, commune of Bethioua, and commune of Arzew. Initial parameter values for epidemic propagation model and variable state are given in Table 2 for the three communes of outbreak of flu.

By varying the values of the variables and parameters, we can simulate many interactions.

\subsection{Visualization System: Results}

In this section, we discuss the different visualization types provided by SIMAS.

\subsubsection{Visualization of Epidemic Evolution}

\subsubsection{Statistics}

The exponential distribution, the $\mathrm{k}$ degree and infection doses can be taken as a stochastic process in which encounters and contamination between infected and susceptible individuals occur randomly.

Since our model is stochastic, we run 5000 iterations for each simulation. The outputs of stochastic SIR agent based model with mean trajectories for each compartment in the experimental population

Table 2. The value of simulation parameters for the three communes

\begin{tabular}{|l|l|l|l|l|l|}
\hline \multicolumn{1}{|c|}{ Commune } & \multicolumn{1}{|c|}{ Beta } & \multicolumn{1}{c|}{ (Gamma) $^{-1}$} & \multicolumn{1}{c|}{ Degree K } & \multicolumn{1}{c|}{ S(0) } & \multicolumn{1}{c|}{ I(0) } \\
\hline Oran & 0.14 & 5 & 10 & 1850 & 105 \\
\hline Bethioua & 0.163 & 6 & 8 & 598 & 12 \\
\hline Arzew & 0.164 & 6 & 9 & 395 & 8 \\
\hline
\end{tabular}


Figure 8. The evolution of SIR within SW epidemic model over time (with and without vaccination)
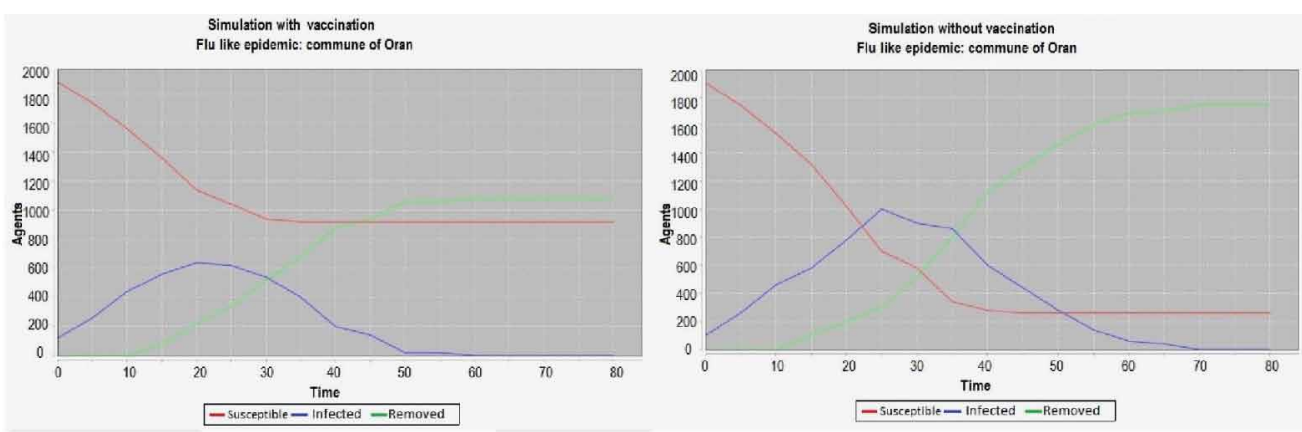

(with and without vaccination) are presented in Figure 8. As an example, we choose the commune of Oran to represent all different results obtained by SIMAS.

Both the two illustrations (with and without vaccination), the three curves have the similar trends. Trace plots provide the number of infected people $\mathrm{I}(\mathrm{t})$ which will increase along with the epidemic spreading, and then it will decrease after the epidemic is brought under control. At the same time, the number of susceptible agents $S(t)$ will decrease because they will move into the Infected class after being infected. The number of recovered $\mathrm{R}(\mathrm{t})$ will be increase after a period of time where individuals begin to leave Infected class. Here, we assume an exponential distribution of infectious periods. During the simulation and changing the parameters of model for each commune, we have observed that model factors: $\beta, \gamma^{-1}$ and $\mathrm{k}$, are the key parameters of the influenza epidemic spread. Thus, we have revealed that reducing significantly the contact number can decrease epidemic transmission more effectively than decreasing the number of transmission rate and latent period. Therefore, when the epidemic outbreaks in the population, we should first reduce exposure to the disease as much as possible, and it is better to limit our activities and relationships with our social or spatial neighbors.

Moreover, the vaccination on the susceptible agents during infection has an effect on epidemic spread and avoidance of infected individuals. As shown in Figure 8, in the simulation without vaccination, we found that the threshold value of influenza epidemic is 1000 cases is day=15 and the end of the epidemic was in day $=70$. However, it was 620 cases in day $=20$ and the end of the flu epidemic was in day $=50$. We calculate, also, the morbidity factor in the three communes. The following results were obtained: Oran (0,004\%), Bethioua (0,033\%) and Arzew (0,005\%). Although the last two communes have a smaller population than the first commune (Oran). The obtained values are explained by the high density of these two communes, especially during the day due to the large gatherings of employees. It should be noted that in these communes is installed the very large industrial area in the field of hydrocarbon exploitation and treatment.

We conclude that threshold of the epidemic diffusion depends on some key parameters, such as average degree distribution of the small-world network, infection doses, transmission rate and infection period.

\subsubsection{Sniffer Agent}

Using the sniffer interface, the decision maker can sniff an agent or a group of agents (interaction history: who infect whom and when the person 'agent' is infected). Every message directed to that agent(s) or coming from that agent(s) is displayed. The decision maker can consult and save all the messages, and he can analyzed later. Since the agent's network is large, we choose a sample of agents to represent the communication between agents. An example of Sniffer agent interface is illustrated in Figure 9. 
Figure 9. An example of Sniffer agent

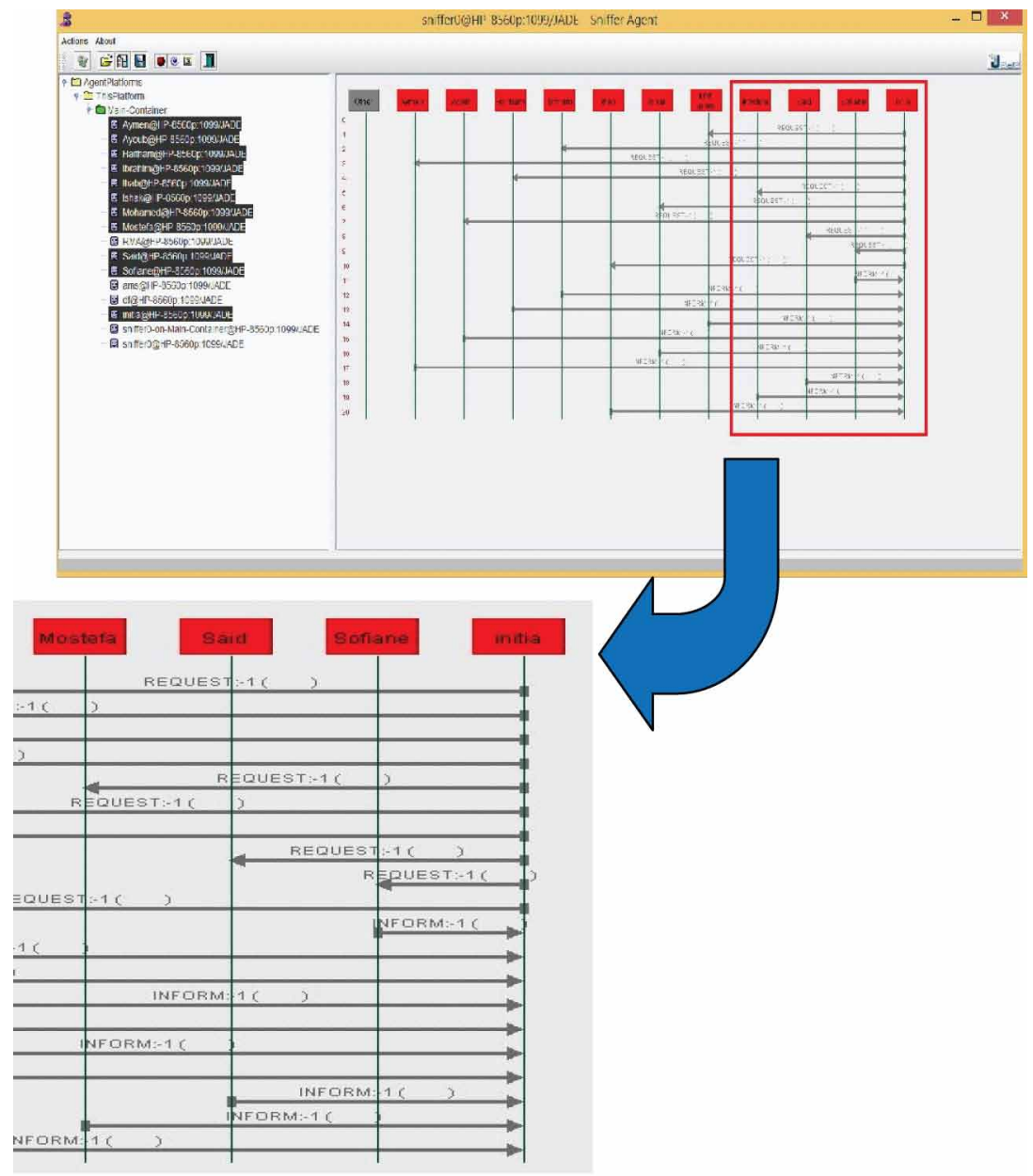

\subsubsection{Visualization of SW Social Network}

We examine the evolution of the epidemic within the agents by representing the network during the infection. We also consider the structure of networks and the role of agents within these networks.

For a clearer and more legible illustration, we took a sample of the infected population ( 30 agents). The Figure 10, presents the risk graph forming a circle (Watts andStrogatz model) for the process of infection in the time for the two cases (with and without vaccination for the commune of Oran).

We choose the same sample to illustrate the SW contact network during the epidemic flu. The obtained results show that the key feature of social network analysis in our study is to understand how relationship between agents changes topological structure of a network according to the preselected parameters of the model. We show how these measures (preselected parameters) might directly influence the progress of a disease modelled on the network. Based on the results, it can be concluded that the vaccination against flu epidemic has a positive impact for reducing the incidence cases and it is recommended in the immunization programs to protect against currently circulating strains. Moreover, the findings provide information to the public health decision maker/policy makers to give additional attention to the spatial planning of effective control measures. 
Figure 10. Agents risk graph during epidemic spread (with and without vaccination)

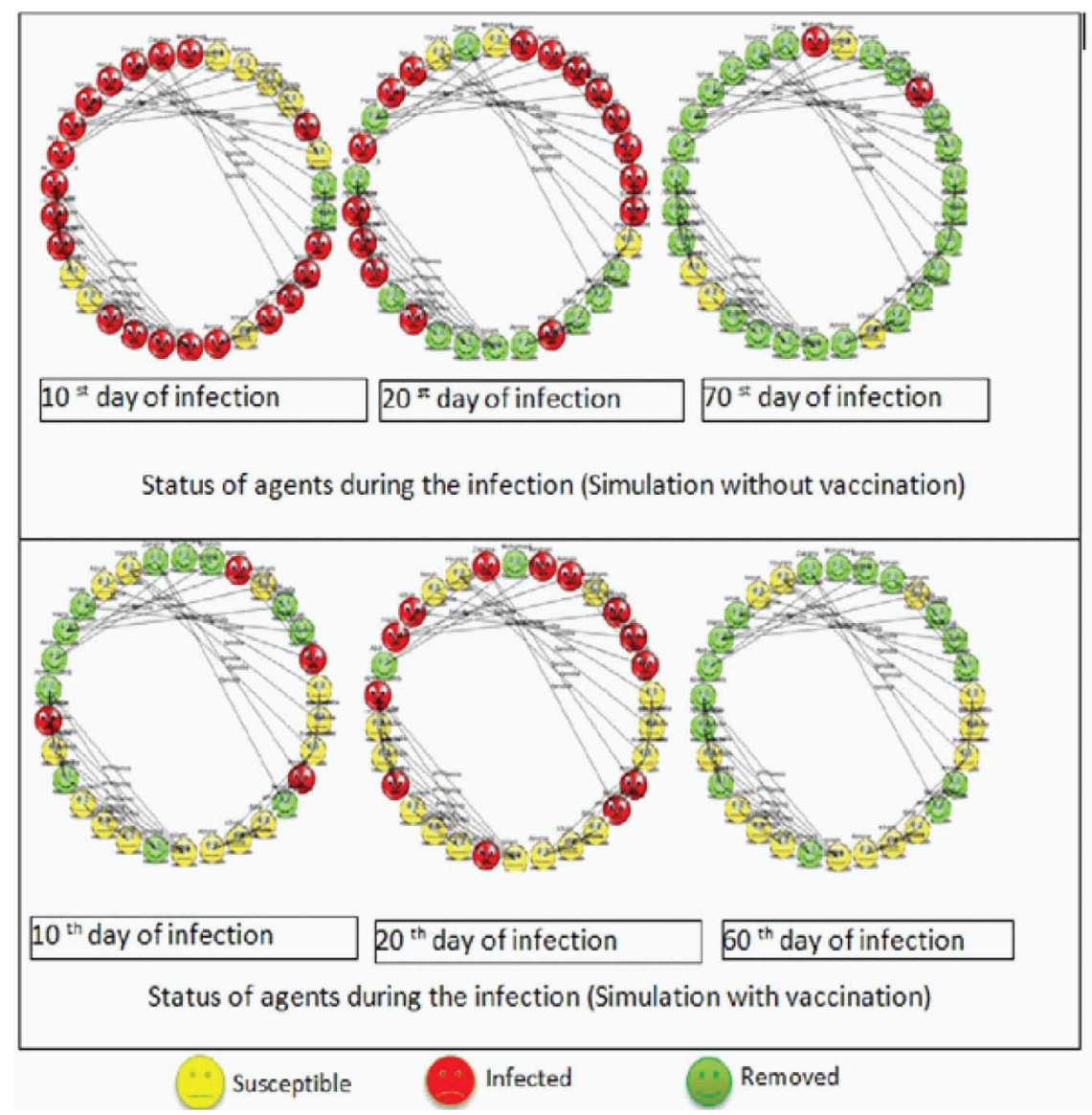

\subsubsection{Visualization of Incidence on a Region Map}

Because spread of infectious disease outbreaks are spatiotemporal phenomena, Geographic Information Systems (GIS) can be an intuitive choice to offer a framework that is well suited for this task. For an easy readability and a clearer understanding of propagation phenomena in all communes, we propose the use of GIS based on agent model to analyze infectious diseases.

The study area shapefile, which incorporates parameters of each commune, was created in ArcGIS 10 using geospatial data obtained from Center for Space Technology (CTS) of Arzew (Oran). The GIS provides a real-time visualization method of spreading epidemics to enhance the visual analytics of the disease outbreak detection. Thematic maps (referred to as data layers in GIS) on incidence numbers of disease have been generated. An incidence map was created to classify the influenza incidence from the lowest level to the highest level. This map is illustrated in Figure 11.

As shown in Figure 11, there is higher incidence of flu in the following communes: Oran, Bethioua, Boutlelis and Arzew, etc. communes have a higher incidence of flu while Tefraoui, Ain el kerma, Senia, El kerma, etc. communes a lower incidence of flu.

The regions of very high risk are part of the Oran sea coast where major infrastructures are installed (hydrocarbons, ammonia production, etc.). Moreover, we conclude that there is a close relation between the incidence of influenza and population density. In some highest incidences communes presented in Figure 11, there are more contact opportunities, because of crowded urban traffic and frequent economic activities. 


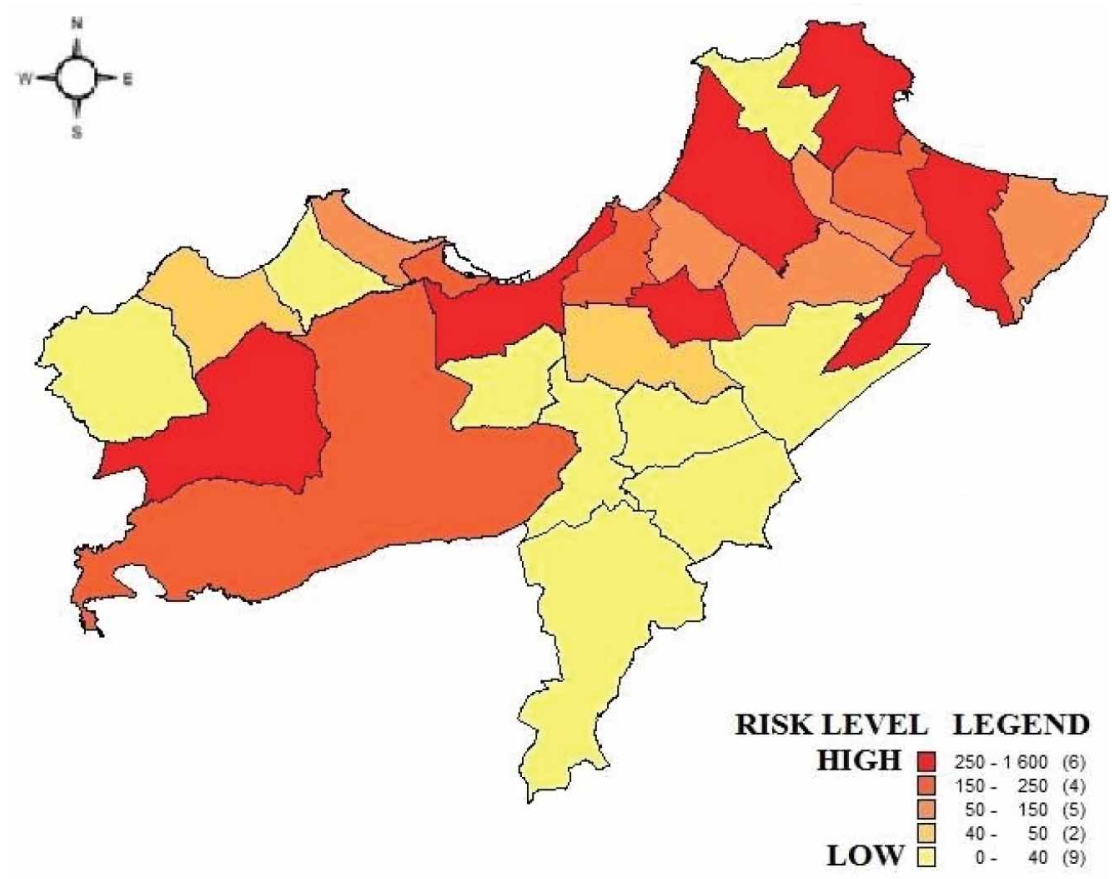

\section{CONCLUSION}

Using multi-agent simulation models to simulate epidemic spread is an emerging trend in research fields. In the current study, we studied a paradigm for modeling and simulation of complex system using agent based model with SIR model and GIS to describe the behavior of agents, simulating it and displaying the risk areas on a map. Multi-agent simulation exploits advantages of agent-based modeling to evaluate the efficiency of infection control measures against the flu infection. We have built a simulation model for infection of influenza and quantitatively assessed infection risk of disease. We, also, discuss the impact of vaccination that remains the best strategy to reduce the risk of becoming seriously ill or even dying, and to reduce the impact of influenza outbreaks on the health system and the economy. We concluded that the topology of network and others factors may have strong effect on reducing the infectious disease transmission. Moreover, preventing measures and control of transmission of influenza virus within human population is important to reduce influenza transmission. The proposed system SIMAS can be readily used in practice and it can be, easily, extended to other locations provide relevant data are available. It can also be used to simulate the spread of any infectious disease that follows the SIR person-to-person transmission dynamics. We believe that SIMAS could eventually be helpful for public health decision makers. It enable them to better understand the impact of different parameters on an outbreak and how vaccinated population can have a major influence on prevention strategies, preparation and building resilient. Thus, it allows health professional to suggest appropriate operational strategies to mitigate or reduce infectious disease spread in human population (keeping a specific distance, wearing masks,lockdown, etc.).

Some limitations of our system are with respect to the run of simulations. In other words, SIMAS works well when we performs simulations with a small number of agents, however, once the number of agents gets large the simulation can become prohibitively slow given the limited computer memory capacity. 
In order to overcome SIMAS functionalities, we propose, in future, to extend our research to study the effect of community structure and to discuss, deeply, the social network analysis in order to identify the best people to vaccinate. Using rich and varied medical and socio-economic data, further work needs to be carried out to study the effect of PCR and others investigations. As known, PCR-based testing is primary measured for early isolation of infected individuals. On a deeper level, some analysis are also needed to discuss the differential diagnosis of infection apart influenza.

\section{ACKNOWLEDGMENT}

The authors would like to thank Public Health Department of Oran and Center for Space Technology of Arzew for their assistance with this study and their helpful collaboration. They would like, also, to thank the Directorate General for Scientific Research and Technological Development (DGRSDT), an institution of the Algerian Ministry of Higher Education and Scientific Research, for their support on this work. 


\section{REFERENCES}

Arduin, H., de Cellès, M. D., Guillemot, D., Watier, L., \& Opatowski, L. (2017). An agent-based model simulation of influenza interactions at the host level: Insight into the influenza-related burden of pneumococcal infections. BMC Infectious Diseases, 17(1), 382. doi:10.1186/s12879-017-2464-z PMID:28577533

Beretta, E., \& Kuang, Y. (2001). Modeling and analysis of a marine bacteriophage infection with latency period. Nonlinear Analysis Real World Applications, 2(1), 35-74. doi:10.1016/S0362-546X(99)00285-0

Cao, G., Cao, L., \& Li, X. (2008, July). The study of epidemic spreading on a mobile multi-agent system with infective expressivity and its control strategy. In Control and Decision Conference, 2008. CCDC 2008 (pp. 4086-4089). IEEE.

Dodds, P. S., \& Watts, D. J. (2004). Universal behavior in a generalized model of contagion. Physical Review Letters, 92(21), 218701. doi:10.1103/PhysRevLett.92.218701 PMID:15245323

Dong, W., \& Heller, K., \& Pentland, A. S. (2012, April). Modeling infection with multi-agent dynamics. In International Conference on Social Computing, Behavioral-Cultural Modeling, and Prediction (pp. 172179). Springer.

Epstein, J. M., Cummings, D. A., Chakravarty, S., Singha, R. M., \& Burke, D. S. (2004). Toward a containment strategy for smallpox bioterror: An individual-based computational approach. Brookings Institution Press.

Eubank, S. (2005). Network based models of infectious disease spread. Japanese Journal of Infectious Diseases, 58(6), S.

Godjedo, T. P. M., Paraiso, M. N., Agbankpe, A. J., Dougnon, T. V., Denon, Y. E., Hidjo, M., \& Bankole, H. (2020). Assessment of the Epidemiological Surveillance System for Bacterial Meningitis in Benin from 2016 to 2018: Case-by-Case Surveillance. WORLD (Oakland, Calif.), 5(3), 53-59.

Hackl, J., \& Dubernet, T. (2019). Epidemic spreading in urban areas using agent-based transportation models. Future Internet, 11(4), 92.

Kano, T., Yasui, K., Mikami, T., Asally, M., \& Ishiguro, A. (2021). An agent-based model of the interrelation between the COVID-19 outbreak and economic activities. Proceedings of the Royal Society of London. Series A, 477(2245), 20200604. PMID:33633491

Kilbourne, E. D. (1978). Opinion: Influenza as a problem in immunology. Journal of Immunology (Baltimore, Md.: 1950), 120(5), 1447-1452. PMID:351051

Kim, L. Y. (2014). Estimating network structure and propagation dynamics for an infectious disease: towards effective vaccine allocation (Doctoral dissertation). Massachusetts Institute of Technology.

Langlois, P., Blanpain, B., \& Daudé, E. (2015). MAGéo, uneplateforme de modélisationet de simulation multiagent pour les sciences humaines. Cybergeo: European Journal of Geography.

Mesgari, M. S., \& Masoomi, Z. (2008). GIS applications in public health as a decision making support system and it's limitation in Iran. World Applied Sciences Journal, 3(1), 73-77.

Moore, K. M., Edgar, B. L., \& McGuinness, D. (2008). Implementation of an automated, real-time public health surveillance system linking emergency departments and health units: Rationale and methodology. Canadian Journal of Emergency Medical Care, 10(2), 114-119. doi:10.1017/S1481803500009817 PMID:18371248

Perez, L., \& Dragicevic, S. (2009). An agent-based approach for modeling dynamics of contagious disease spread. International Journal of Health Geographics, 8(1), 50. doi:10.1186/1476-072X-8-50 PMID:19656403

Ritter, T. A. (2002, June). LEADERS: Lightweight epidemiology advanced detection and emergency response system. In Chemical and Biological Sensing III (Vol. 4722, pp. 110-121). International Society for Optics and Photonics.

Toma, B., Bénet, J. J., Dufour, B., Eloit, M., Moutou, F., \& Sanaa, M. (1991). Glossary of animal epidemiology. Editions du Point Vétérinaire. 
Verdasca, J., Da Gama, M. T., Nunes, A., Bernardino, N. R., Pacheco, J. M., \& Gomes, M. C. (2005). Recurrent epidemics in small world networks. Journal of Theoretical Biology, 233(4), 553-561. doi:10.1016/j. jtbi.2004.10.031 PMID:15748915

Watts, D. J., \& Strogatz, S. H. (1998). Collective dynamics of 'small world' networks. Nature, 393(6684), 440.

Xia, H., Chen, J., Marathe, M., \& Mortveit, H. S. (2011, March). Synthesis and refinement of detailed subnetworks in a social contact network for epidemic simulations. In International Conference on Social Computing, Behavioral-Cultural Modeling, and Prediction (pp. 366-373). Springer. doi:10.1007/978-3-642-19656-0_50

Xiao, H., Tian, H., Shao, L., Zhao, J., \& Xu, J. Z. (2010, October). Spatio-temporal Simulation of Epidemiological SIQR Model Based on the Multi-Agent System with Focus on Influenza A (H1N1). In International Symposium on Intelligence Computation and Applications (pp. 180-189). Springer. doi:10.1007/978-3-642-16388-3_20

Yang, F., Yang, Q., Liu, X., \& Wang, P. (2015). SIS evolutionary game model and multi-agent simulation of an infectious disease emergency. Technology and Health Care, 23(s2), S603-S613. doi:10.3233/THC-150999 PMID:26410529

Younsi, F. Z., Hamdadou, D., \& Boussaid, O. (2015). A Surveillance and Spatiotemporal Visualization Model for Infectious Diseases using Social Network. International Journal of Decision Support System Technology, 7(4), 1027-1046.

Younsi, F. Z., Hamdadou, D., \& Boussaid, O. (2017). Integration of Simulation System in Data Warehouse. Proc. 6th International Conference on Systems and Control, 43-48.

Younsi, F. Z., Hamdadou, D., \& Boussaid, O. (2019a). A surveillance and spatiotemporal visualization model for infectious diseases using social network. In Censorship, Surveillance, and Privacy: Concepts, Methodologies, Tools, and Applications (pp. 1027-1046). IGI Global.

Younsi, F. Z., Hamdadou, D., \& Chakhar, S. (2019b). A multicriteria spatiotemporal system for influenza epidemic surveillance. In Technological Innovations in Knowledge Management and Decision Support (pp. 176-202). IGI Global.

Zhang, M., Verbraeck, A., Meng, R., Chen, B., \& Qiu, X. (2016). Modeling spatial contacts for epidemic prediction in a large-scale artificial city. Journal of Artificial Societies and Social Simulation, 19(4), 3. doi:10.18564/ jasss. 3148

\section{ENDNOTES}

http://www.dsp-oran.dz/

https://www.who.int/

Fatima-Zohra Younsi is Senior lecturer in computer science at department of Science and Technic in University of Abdelhamid Ibn Badis, Algeria, where she has been since 2009. She received her engineer degree in computer science from the University of Oran Es-Senia, Algeria in 2004. She also obtained her PhD degree in 2016 from the University of Oran1, Algeria and the University of Lyon 2, France. Her current research interests span both data analysis and epidemic surveillance. Much of her work has been on improving the understanding, design, and performance of decisional information systems, mainly through the application of Geographic Information System (GIS), social network analysis, Business intelligence (BI), Artificial Neural Network and mathematical modeling.

Djamila Hamdadou received her Engineering degree in Computer Science and her Master of Science degree from the Computer Science Institute in 1993 and 2000, respectively. She also obtained her doctorate in 2008. She received her PHD in 2012 from the Computer Science Department. She is specialized in Artificial Intelligence, Decision Support Systems, Multi Criteria Analysis, Collaborative and Spatio Temporel Decisional Systems and Business Intelligence. She is a Professor at the University Oran 1 in Algeria where she leads the research team "Artificial Intelligence Tools at the service of Spatio-Temporal and Medical Decision Support" at the laboratory of computer science of Oran (LIO). 